\title{
Irreducible Atlanto-Axial Dislocation in Neglected Odontoid Fracture Treated with Single Stage Anterior Release and Posterior Instrumented Fusion
}

\author{
Rishi Anil Aggarwal ${ }^{1}$, Ashok Keshav Rathod ${ }^{2}$, Kshitij Subhash Chaudhary ${ }^{3}$ \\ ${ }^{I}$ Department of Orthopaedics, Seth G.S. Medical college and K.E.M. Hospital, Maharashtra, India \\ ${ }^{2}$ Department of Orthopaedics, Lokmanya Tilak Municipal Medical College and General Hospital, Maharashtra, India \\ ${ }^{3}$ Department of Orthopaedics, Sir HN Reliance Hospital and Research Center, Maharashtra, India
}

It is a well-know fact that type 2 odontoid fractures frequently go into nonunion. If left untreated, patients may develop irreducible atlantoaxial dislocation (AAD). We describe the surgical management of two patients with neglected odontoid fractures and irreducible AAD treated with single stage anterior release followed by posterior instrumented fusion. Both patients presented with history of neglected trauma and progressive myelopathy. Traction under anesthesia failed to achieve reduction of AAD. Anterior release was done by trans-oral approach in one patient and retrophayngeal approach in the other. Posterior fixation was performed with transarticular screws in both the patients. Both patients had full neurological recovery and demonstrated fusion at follow-up. Anterior release followed by posterior instrumented correction may be an effective alternative to the traditional means of treating irreducible dislocations associated with neglected odontoid fractures.

Keywords: Atlantoaxial joint; Dislocation; Atlantoaxial dislocation; Neglected; Odontoid process

\section{Introduction}

Neglected spinal injuries are frequently encountered in the developing world. Overlooked diagnoses, poor accessibility to healthcare and socio-economic factors are the major reasons for such occurrences [1]. Often these injuries cannot be treated with conventional surgical techniques and require innovative ideas. As neglected spinal injuries are infrequently discussed in literature, we would like to present two cases of neglected odontoid fractures with irreducible dislocation and myelopathy. These cases were treated with anterior release of contractures and/or structures blocking the reduction, followed by posterior $\mathrm{C} 1-\mathrm{C} 2$ fusion in single stage. We present the surgical technique and the outcome of these patients.

\section{Technical Note}

\section{Case 1}

A 24-year-old man presented to us with neck pain and progressive difficulty in walking with weakness in the

\footnotetext{
Received Aug 7, 2015; Revised Aug 8, 2015; Accepted Aug 25, 2015

Corresponding author: Rishi Anil Aggarwal

Department of Orthopaedics, Seth G.S. Medical College and K.E.M. Hospital, Parel, Mumbai - 400012, Maharashtra, India

Tel: +91-22-410-7463, Fax: +91-22-410-7463, E-mail: ris1987@gmail.com
} 
right hand since one month. One year ago he had injured his neck in a road traffic accident after a fall off a bike. He did not seek medical attention and claims that he did not
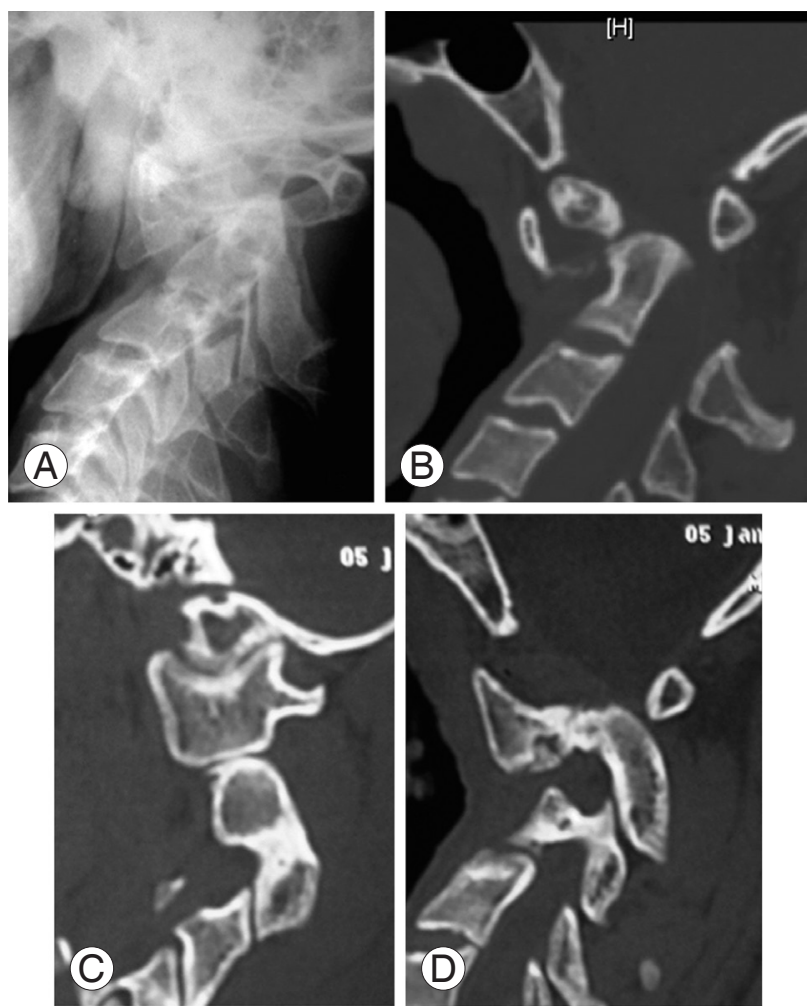

Fig. 1. (A) Lateral X-ray of cervical spine showing atlantoaxial dislocation with localized kyphosis. (B) Computed tomography (CT) scan showing nonunion of type II dens fracture with atlantoaxial dislocation. and clivus canal angle of $114^{\circ}$. (C) Right sided parasagittal CT scan showing normal C1-2 joint. (D) Left sided parasagittal CT scan showing dislocated and arthritic C1-2 joint. have any weakness at that time. He had torticollis with a right-sided head tilt, spasticity and exaggerated deep tendon reflexes in all extremities. Right hand grip was weak. Rest of the muscle groups had normal motor power. Sensations were preserved and the plantar response was extensor bilaterally.

Radiographs revealed rotatory atlanto-axial dislocation with anterior subluxation of atlas (Fig. 1A), which did not reduce on dynamic views. Computed tomography (CT) revealed nonunion of type II dens fracture (Fig. 1B). There was significant $\mathrm{C} 1-2$ kyphosis with a clivuscanal angle of $114^{\circ}$. The right sided C1-C2 joint was well aligned, however, the left side sided joint was dislocated and arthritic (Fig. 1C, D). This was probably the principal reason for the irreducibility. Magnetic resonance imaging (MRI) revealed severe cord compression at the craniovertebral junction with a cervico-medullary angle of $126^{\circ}$.

Skeletal traction was progressively increased up to 10 $\mathrm{kg}$ over 2 days preoperatively, but this failed to improve the alignment. The patient was taken for surgery and following general anesthesia and complete muscle paralysis, sustained skeletal traction up to $15 \mathrm{~kg}$ was given for ten minutes. This led to slight improvement in C1-2 kyphosis, but anterior translation of $\mathrm{C} 1$ could not be corrected (Fig. $2 \mathrm{~A})$.

\section{1) Surgical technique}

The neck was positioned in hyperextension under cervical traction. A transnasal catheter sutured to the uvula was used to retract the soft palate. Crockard transoral
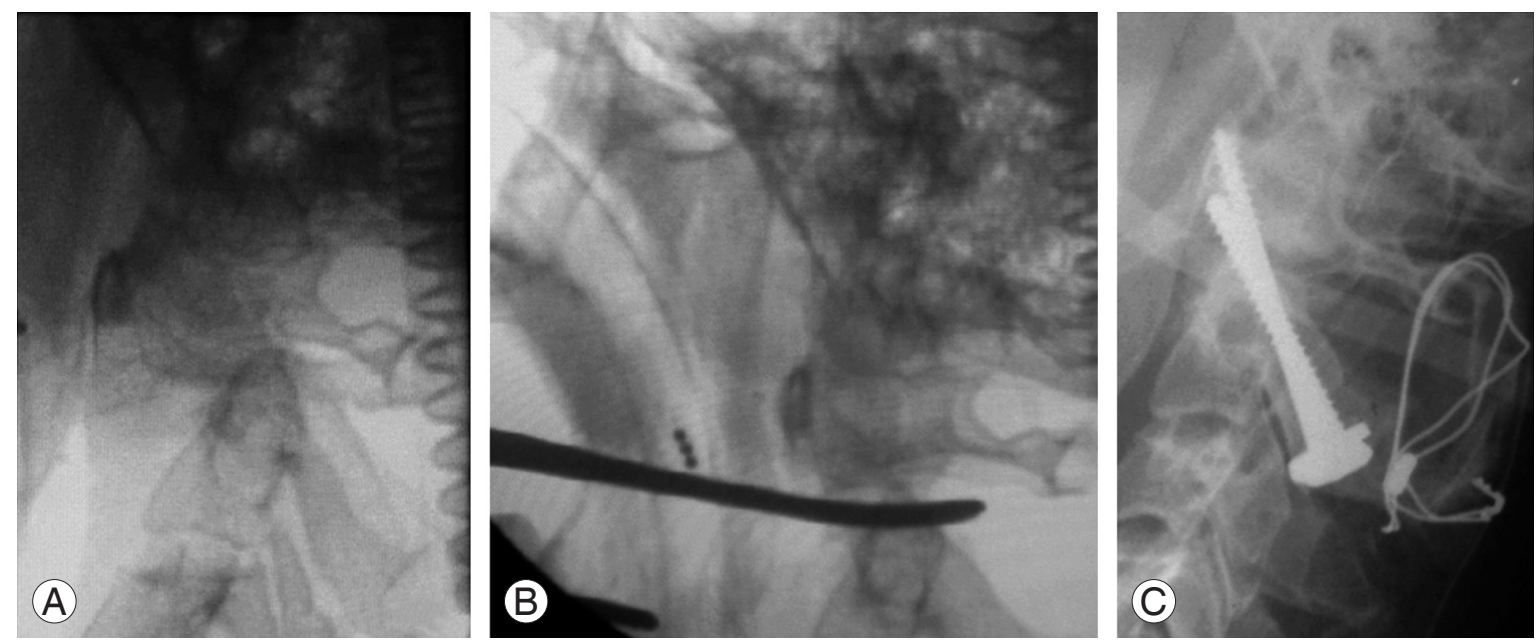

Fig. 2. (A) Fluoroscopic image after giving traction under anesthesia showing irreducible atlantoaxial dislocation. (B) Blunt instrument used to release C1-2 intra-articular adhesions following opening of joint capsule during anterior release. (C) Immediate postoperative X-ray. 
retractor system provided a standard transoral exposure. The longus colli, longus capitis muscles and the anterior longitudinal ligament were transversely divided along the inferior border of the $\mathrm{C} 1 \mathrm{arch}$. The anterior capsule of the atlantoaxial joint was incised. A 5-mm periosteal elevator was used to distract the atlantoaxial joint taking care to remain within the lateral edge (Fig. 2B). This released any intra-articular adhesions.

Following anterior release, the patient was carefully turned prone while maintaining skeletal traction. Posterior exposure of atlantoaxial region was then performed. A sublaminar wire was passed under $\mathrm{C} 1$ posterior arch. This was used to give a posteriorly directed force to achieve and maintain anatomical reduction while definitive fixation was performed. Trans-articular screws were used for fixation and autologous posterior iliac crest bone graft used for fusion (Fig. 2C).

\section{2) Postoperative management}

The patient was mobilised soon after surgery in a Philadelphia collar. Nasogastric tube feeding was continued for 7 days till the incision on posterior pharyngeal wall healed. Patient had a complete neurological recovery at the 3-month follow up visit. Radiographs at 24-month follow-up revealed maintained C1-2 alignment and good consolidation of the posterior bone graft (Fig. 3A). A CT at one year showed anatomical C1-2 alignment with a clivus-canal angle of $145^{\circ}$ (Fig. 3B).

\section{Case 2}

A 9-year-old male child presented with gradually progres-
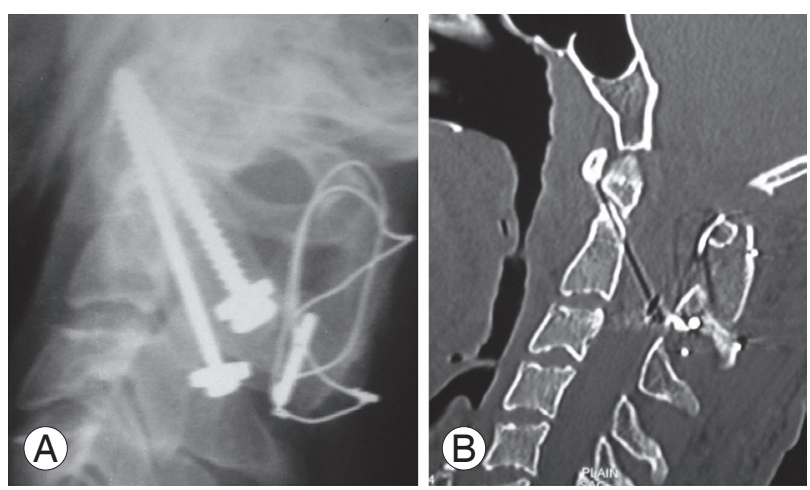

Fig. 3. (A) X-ray at follow-up showing normal C1-2 alignment with C1-2 posterior fusion. (B) Computed tomography scan at follow up showing anatomical alignment of craniovertebral junction with a clivus-canal angle of $145^{\circ}$. sive weakness in both hands and difficulty in walking of 3 weeks duration. He had a history of fall from height of approximately 10 feet, six months ago followed by injury to his neck for which no treatment was taken. On examination he had weakness of bilateral handgrip with spasticity and exaggerated deep tendon reflexes in all extremities. He had grade 4 to 5 strength in the rest of the muscle groups without any sphincter dysfunction. Sensations were intact to light touch.

Radiographs, including dynamic views, revealed irreducible atlantoaxial dislocation (Fig. 4A). Preoperative skeletal traction up to $6 \mathrm{~kg}$ did not achieve reduction (Fig. 4B). CT scan revealed type II dens fracture in nonunion. There was some new bone formation at the fracture site, which was probably one of the contributing factors preventing reduction (Fig. 4C). MRI revealed severe cord compression at the cranio-vertebral junction. Traction under anesthesia and complete muscle relaxation failed to achieve reduction.

\section{1) Surgical technique}

The patient had restricted mouth opening, hence anterior release was done via retropharyngeal approach. An oblique incision was taken between the thyroid notch and hyoid cartilage extending from midline to the lateral border of sternocleidomastoid muscle. Platysma was cut in line with the incision. The hypoglossal nerve runs parallel to the tendon of digastric muscle. It was dissected and protected. The sternocleidomastoid muscle and carotid sheath were retracted laterally. The strap muscles, hyoid and pharynx were carefully retracted medially to expose the retropharyngeal space. Following this the arch of $\mathrm{C} 1$ was palpated. A blunt instrument was placed on the arch of $\mathrm{C} 1$ and position was confirmed under fluoroscopy. Similar releases were carried out as described for Case 1. Fluoroscopic image after anterior release showed satisfactory alignment (Fig. 4D). The posterior instrumentation and fusion (Fig. 4E, 4F) was done using transarticular screws. A sublaminar wire was passed under $\mathrm{C} 1$ posterior arch. This was used to give a posteriorly directed force to maintain reduction while trans-articular screws were passed (Fig. 4E).

\section{2) Postoperative management}

Postoperative recovery was uneventful except for dysphagia that required a course of intravenous dexamethasone and nasogastric feeding for 3 weeks. The patient had a 



Fig. 4. (A) Lateral X-ray of cervical spine showing atlantoaxial dislocation (AAD). (B) Lateral X-ray following traction in ward showing irreducible AAD. (C) Computed tomography scan showing nonunion type II dens fracture. Some new bone formation is seen at the fracture site, which was probably one of the contributing factors preventing reduction. (D) Anatomical C1-2 alignment following anterior release. (E) A sublaminar wire was passed under C1 posterior arch which was used to give a posteriorly directed force to maintain reduction while trans-articular screws were passed. (F) Postoperative X-ray.

complete neurological recovery at 3 months follow-up and $\mathrm{X}$-rays showed bridging bone across posterior elements of $\mathrm{C} 1-\mathrm{C} 2$ at one year follow up.

\section{Discussion}

It is a well-known fact that type 2 odontoid fractures frequently go into nonunion. The reported rate in literature varies between $26 \%$ to $76 \%$ [2]. The instability caused by the fracture along with poor vascularity of this region, as it is enveloped in synovium, is proposed as a probable explanation of this occurrence. If left untreated, $\mathrm{C} 1$ eventually loses support from the $\mathrm{C} 2$ superior articular process. With time, the tissues in the concavity of the deformity, which is ventral to the craniovertebral area, contract and shorten. The longs colli, longus capitis, anterior joint capsule and the anterior longitudinal ligaments are all in the concavity of the deformity (C1-C2 kyphosis) and undergo contracture preventing complete reduction.

In addition to soft tissue contractures, bony changes may also play a role in irreducibility. The articular surfaces of C1-2 may become gradually more vertical and arthritic preventing the reduction of atlas over axis. Furthermore, new bone formation between dens and body of axis may prevent posterior translation of dens hence preventing reduction of C1-2 joint. Arthritis of the $\mathrm{C} 1-\mathrm{C} 2$ joint (Case 1) and new bone formation at the fracture site (Case 2) were probably the reasons for irreducibility in our patients. In 
rare cases malunion of the odontoid has been reported as the cause for irreducibility.

Traditionally irreducible atlantoaxial dislocations have been treated with transoral odontoidectomy followed by posterior fusion [3-5]. With technological advances in microsurgical techniques, the safety of transoral odontoidectomy has improved substantially. However, it is still technically demanding and carries a small but significant risk of incidental dural leak that may lead to meningitis. During transoral odontoidectomy no attempt is made to correct the atlantoaxial kyphosis or the rotatory malalignment. This may result in swan neck deformity in the cervical spine or residual torticollis respectively [6].

Posterior atlantoaxial joint release and distraction is an alternative described by Goel [7]. Although this procedure works well in the less severe irreducible dislocation, we have found it considerably difficult in achieving anatomical correction in patients with severe dislocation. In a patient with complete spondyloptosis of the $\mathrm{C} 1$ over $\mathrm{C} 2$ that is irreducible, we have found it difficult to access the $\mathrm{C} 1-\mathrm{C} 2$ joint from the posterior approach without sacrificing the $\mathrm{C} 2$ superior articular facet. This is similar to performing sacral dome osteotomy done to gain access in the spondyloptotic L5-S1 disc space in high-grade spondylolisthesis. In our experience, excessive drilling in this area results in loss of the $\mathrm{C} 2$ superior articular facet and its underlying bone making it difficult to place a $\mathrm{C} 2$ pedicle screw safely. Moreover, this technique of posterior distraction of facets fails to address the primary tether (contracted longus colli, longus capitis and the anterior facet capsule) in chronically dislocated joints.

Wang et al. [6] in 2007 showed that anterior transoral release of irreducible atlantoaxial dislocation followed by posterior instrumented fusion can achieve excellent alignment even in severe dislocations. Several authors have reported successful application of this technique to treat irreducible dislocations [8-10]. There are several advantages of this technique over transoral odontoidectomy. An anatomical reduction can be achieved preventing subaxial swan-neck deformity. Torticollis can be corrected. Transoral dural injuries and its associated morbidity can be avoided. The release of $\mathrm{C} 1-\mathrm{C} 2$ joints anteriorly also makes the posterior reduction maneuver technically easier. We propose that in patients in whom transoral odontoidectomy is planned, an initially release procedure under fluoroscopy should be attempted. If the release is unable to achieve adequate correction then the surgery can proceed to odontoidectomy.

There are some reports of neglected odontoid fractures treated with this method [4,11-13]. Anterior release of contracted structures can be performed trans-orally or via retropharyngeal approach. Microendoscopic anterior release and video-assisted transcervical anterior release have also been described in the literature. Most surgeons prefer posterior fixation following anterior release; however, $\mathrm{Wu}$ et al. [11] described a technique of microendoscopic anterior release and anterior transarticular screw fixation for patients with irreducible atlantoaxial dislocation.

We used the transarticular screw fixation for posterior $\mathrm{C} 1-\mathrm{C} 2$ fusion. An alternative technique is to use the Goel Harms C1 lateral mass and C2 pedicle screw fixation. There are definite advantages of the latter technique over transarticular screws. Particularly, in the cases presented, the Goel Harms technique would have been technically easier as this method does not require anatomical reduction of $\mathrm{C} 1-\mathrm{C} 2$ joint before fixation. Rotational malalignment is also easier to correct with this technique.

In conclusion, neglected nonunion of odontoid fracture may lead to irreducible dislocation. Anterior release followed by posterior instrumented correction may be an effective alternative to the traditional means of treating irreducible dislocations.

\section{Conflict of Interest}

No potential conflict of interest relevant to this article was reported.

\section{References}

1. Sengupta DK. Neglected spinal injuries. Clin Orthop Relat Res 2005;(431):93-103.

2. Pal D, Sell P, Grevitt M. Type II odontoid fractures in the elderly: an evidence-based narrative review of management. Eur Spine J 2011;20:195-204.

3. Jain VK. Atlantoaxial dislocation. Neurol India 2012; 60:9-17.

4. Jain VK, Behari S, Banerji D, Bhargava V, Chhabra DK. Transoral decompression for craniovertebral osseous anomalies: perioperative management dilemmas. Neurol India 1999;47:188-95.

5. Tuite GF, Veres R, Crockard HA, Sell D. Pediatric transoral surgery: indications, complications, and long-term outcome. J Neurosurg 1996;84:573-83. 
6. Wang C, Yan M, Zhou HT, Wang SL, Dang GT. Open reduction of irreducible atlantoaxial dislocation by transoral anterior atlantoaxial release and posterior internal fixation. Spine (Phila Pa 1976) 2006;31:E30613.

7. Goel A. Treatment of basilar invagination by atlantoaxial joint distraction and direct lateral mass fixation. J Neurosurg Spine 2004;1:281-6.

8. Hao D, He B, Zheng Y, Zhang Z. Single stage anterior release and sequential posterior fusion for irreducible atlantoaxial dislocation. J Spinal Disord Tech 2013 Jan 5 [Epub]. http://dx.doi.org/10.1097/ BSD.0b013e31826be885

9. Hao DJ, He BR, Wu QN. One-stage anterior release and reduction with posterior fusion for treatment of irreducible atlantoaxial dislocation. Orthop Surg 2009;1:305-10.
10. Wang S, Wang C, Yan M, Zhou H, Dang G. Novel surgical classification and treatment strategy for atlantoaxial dislocations. Spine (Phila Pa 1976) 2013; 38:E1348-56.

11. Wu YS, Chi YL, Wang XY, et al. Microendoscopic anterior approach for irreducible atlantoaxial dislocation: surgical techniques and preliminary results. J Spinal Disord Tech 2010;23:113-20.

12. Liu T, Li F, Xiong W, et al. Video-assisted anterior transcervical approach for the reduction of irreducible atlantoaxial dislocation. Spine (Phila Pa 1976) 2010;35:1495-501.

13. Zhai MY, Wang CP, Liu F, Liu YQ, Zhang P. The efficacy of pedicle screw instrument in treatment of irreducible atlantoaxial dislocation. Arch Orthop Trauma Surg 2015;135:1193-9. 